\title{
Pathology Growth Model Based on Particles
}

\author{
Raimundo Sierra ${ }^{1}$, Michael Bajka $^{2}$, and Gábor Székely ${ }^{1}$ \\ 1 Computer Vision Group, ETH Zürich, Switzerland \\ \{rsierra, szekely\}@vision.ee.ethz.ch \\ 2 Clinic of Gynecology, Dept. OB/GYN, University Hospital of Zürich, Switzerland
}

\begin{abstract}
Virtual reality based surgical simulators offer the possibility to provide training on a wide range of findings of different pathologies. Current research aims at a high fidelity hysteroscopy simulator. Different methods for the generation of pathologies have been investigated to realize the first surgical simulator that challenges the trainee with a new scene in every training session. In this paper, a particles-based tumor growth model is presented that overcomes different limitations of previous approaches. It allows for a realistic generation of both polyps and myomas protruding to different extents into the uterine cavity. The model incorporates several biological as well as mechanical factors, which influence the growth process and thus the appearance of the pathologies.
\end{abstract}

\section{Introduction}

The increasing number of endoscopic interventions contrasts with the available training possibilities for future surgeons. Nowadays, basic training for the correct handling of the instruments is performed on bell peppers. Already the next training step is a real intervention under the supervision of an experienced surgeon, introducing an inevitable risk for the patient. Surgical training simulators have the potential to provide a much higher fidelity than today's rudimentary training possibilities without introducing any risk for the patient. Even critical situations could be recreated turning the simulator into an attractive training option also for experienced physicians. The computational power that became available in the last few years enabled the development of many different simulator systems [137. There is consensus on the possible benefits of such virtual reality based training systems, provided that they can overcome some of the current limitations. One major restriction is the use of a single static organ model, whereas the introduction of variable surgical cases is mandatory for effective training. The goal of our current research is a high fidelity hysteroscopy simulator that provides new training scenes as required. This task entails both the generation of variable anatomical models of the healthy organ as well as the incorporation of different pathologies found therein.

Statistical anatomical models, such as the ones employed for the incorporation of prior anatomical knowledge into the segmentation process 441] offer an appealing way to handle the variability of healthy human anatomy within the organ models used for simulation. Such an approach is no longer feasible for the 
generation of pathologies, as the enormous range of manifestations as well as the amount of different pathologies prevent any reasonable statistical analysis. In addition, available non-invasive imaging procedures such as MRI and ultrasonic imaging do not offer the quality required to reconstruct the investigated pathologies accurately.

So far, a cellular automaton able to grow leiomyomas 12 and a skeleton based design method for both polyps and leiomyomas 11 have been presented for the generation of pathologies fulfilling the requirements of a sophisticated surgical training simulator. The main requirement for any pathology generation algorithm is the fully automatic generation of realistic shapes so that a physician can easily specify the desired pathology in medical terms and obtain a reasonable model without any further interaction. The cellular automaton is based on a set of rules that model the genesis of leiomyomas. The skeleton based method aims at a direct design of the desired structures and is based on the physician's experience and visual inspection of hysteroscopic images. Both methods are able to generate visually satisfactory results. The cellular automaton is, however, limited to model spherical growth patterns and is bound to stiff tumors that grow almost independently of the surrounding tissue. While these constraints hold for myomas, they are certainly not true for polyps, as their shape is strongly influenced by the form of the uterine cavity. The skeleton based procedure is a design tool building on appearance and does not model any gestation process. It is therefore not possible to introduce more biological knowledge into the model, e.g. the discrimination of a stiffer inner core in case of myomas. For both approaches an additional processing step is required to merge the pathology with the healthy organ.

The presented alternative simulates, similar to the cellular automaton approach, the genesis of the pathologies under scrutiny by using particles. Modeling the pathologies by their genesis entails many observation from biology and medicine and can thus compensate for the knowledge otherwise obtained by statistical analysis. The current model is a very promising approach that can handle different pathologies in a consistent framework and overcome the limitations of previous models.

\section{Polyp and Myoma Formation}

The pathologies implemented are leiomyomas and polyps protruding to different extents into the uterine cavity. The following paragraphs briefly describe the relevant properties related to the proposed algorithm. More in depth descriptions are available in previous publications or in dedicated literature [5]8].

Myoma Formation. Uterine leiomyomas are well-circumscribed, non-cancerous tumors arising from the myometrium of the uterus. They are found in $25-40 \%$ of women in their childbearing years and are classified by their location in the uterus. In hysteroscopy, submucosal leiomyomas, both sessile and pedunculated, protruding to different extents into the cavity, are visible and treatable. As a myoma is composed of very dense fibrotic tissue, it has a much stronger tendency 
to keep its shape than any of the surrounding tissues. Therefore, the myoma will be able to grow almost independently from its surroundings and keep a spherical shape. The size of a myoma can range from a few millimeters up to several decimeters.

Polyp Formation. Polyps originate as focal hyperplasias of the endometrium and develop into benign, localized overgrowth of endometrial tissue covered by epithelium and containing a variable amount of glands, stroma, and blood vessels. These components make them - in contrast to myomas - very soft and explains the influence of the cavity's shape on their form. Polyps may be broad-based and sessile, pedunculated, or attached to the endometrium by a slender stalk. Furthermore, they vary in size from one millimeter to a mass that fills and expands the entire uterine cavity. They rarely exceed $1.5 \mathrm{~cm}$ in diameter. Large polyps may extend down the endocervical canal or may even protrude into the vagina, being visible on physical examination. They may originate anywhere in the uterine cavity, but occur mostly in the fundus, usually in the cornual region.

\section{Pathology Growth Model}

The focus of the pathology growth model is clearly on the generation of macroscopic findings, which can be provided to the simulator. While ideally all processing steps are based on biological truth, current knowledge is far from providing all details for a complete simulation. Furthermore, a computational simulation requires a trade-off between tractability and accuracy of the model. In a macroscopic model a single particle has to represent a collection of cells rather than a single cell. In the following, the concepts used in the pathology growth model are discussed.

Particles $p$ are used as entities in a three-dimensional space which carry conserved attributes (e.g. size represented by a radius $r$, mass $m$ ) and variable attributes (e.g. position $\boldsymbol{x}_{p}$ and forces $F_{p}$ ). The subscript denotes the type of the particle and the superscript the index.

The interaction between the particles is motivated by the current knowledge of morphogenesis: During morphogenesis, mechanical forces generated by the dynamic rearrangements of cell-cell contacts and the cytoskeleton modulate the changes in cell shape and motility that transform uniform sheets of cells into specialized three-dimensional structures. As morphogenesis proceeds, groups of cells must remain cohesive, while selectively disassembling other intercellular and substratum connections [3].

The particles do not have fixed connections to their neighbors. Each particle interacts with its current neighbors based on a force profile, which relates the distance $r_{i j}$ between the particles $p^{i}$ and $p^{j}$ to a force $F$. As all particles are radial-symmetric, the function is independent of the direction of $\boldsymbol{r}_{i j}$. Theoretically, any function $f$ relating the distance $r$ to a force $F(r), f: \Re \rightarrow \Re$ can be specified. The group of monotonically decreasing and positive functions will only be able to model repulsion of particles thus increasing the inter-particle distance. To represent the tensile and compressive forces acting on cells the $\Lambda_{2}$ 
function, defined by:

$$
\Lambda_{2}: \quad F(r)=\left\{\begin{aligned}
0 & : \quad r>\frac{3}{2} r_{o} \\
\frac{1}{2}\left(1-\frac{r}{r_{0}}\right)\left(2-\frac{r}{r_{0}}\right) & : \quad \frac{1}{2} r_{o} \leq r \leq \frac{3}{2} r_{o} \\
1-\left(\frac{r}{r_{0}}\right)^{2} & : \quad 0 \leq r<\frac{1}{2} r_{o}
\end{aligned}\right.
$$

was selected [2]. $r_{0}$ is the closest equilibrium distance between two particles where $F\left(r_{0}\right)=0$. This function has discontinuities at $r=\frac{1}{2}$ and $r=\frac{3}{2}$, a limited amplitude and range of influence, and both repulsive and attractive ranges of $r$. Particles interacting with a $\Lambda_{2}$ force profile will arrange themselves in a regular grid with a preferred inter-particle distance $r_{0}$.

Growth models of tissues have to be based on static equilibriums rather then on dynamic simulations. There is no meaningful interpretation of a cell velocity or even acceleration when investigating the behavior of one tissue under the influence of a second tissue, e.g. a tumor. This observation contrasts with the simulation of organs when performing surgical tasks like cutting. Therefore, all simulation steps performed in the current algorithm aim at a new equilibrium state after every growth iteration. Updating the particle's position is thus based on a quasi-static solution of the Euler integration scheme:

$$
\boldsymbol{x}^{t+h}=\boldsymbol{x}^{t}+\frac{h^{2}}{m} \boldsymbol{F}^{t}
$$

that can easily be obtained by selecting a high damping coefficient $\gamma=\frac{2 m}{h}$ in the classical Euler differential equation solver.

Three different types of particles are used in the algorithm. The myometrium or healthy tissue is represented by a constant number of particles $p_{\text {tissue }}$ distributed randomly in an initial configuration. The pathology consists of a single initial particle $p_{\text {tumor }}$ with similar properties to the tissue particles. The basic difference is that the tumor particles have the ability to divide, modeling the increased mitotic index of tumor cells.

The endometrium covers any tissue at any time, as it is a highly reactive tissue. It is modeled by particles $p_{\text {endo }}$ which are additionally interconnected by triangles to build an impermeable membrane for all other particles. The edges of the triangles are represented by springs to allow for limited deformations of the surface.

\section{Algorithm Outline}

The algorithm consists of a growth loop, which is iterated until the desired final stage of the tumor is reached, which is specified by the user in terms of size of the pathology. The initial location of the first tumor particle $p_{\text {tumor }}^{0}$ can be selected interactively. In every iteration the following operations are performed:

1. Divide one tumor particle

2. Adapt tissues
3. Adapt surface

4. Surface fairing 
Divide one tumor particle. Tumor cells have increased proliferative rates causing the abnormal growth. This is the only active process in the algorithm, in contrast to the following steps that are reactions to the particle division. Two different rules are used, depending on the type of pathology. As mentioned, the polyp originates from a local overgrowth of the endometrium. Therefore one particle belonging to the endometrium is marked as initial tumor $p_{\text {tumor }}^{0}$ and this particle will be divided in every iteration:

$$
\mathcal{R}_{\text {dividepolyp }}: \begin{array}{rlrl}
p_{\text {tumor }}^{0} & \rightarrow p_{\text {tumor }}^{0}+p_{\text {tumor }}^{n+1} & & n=\left|p_{\text {tumor }}\right| \\
\boldsymbol{x}_{p_{\text {tumor }}^{n+1}}^{n+1}=\boldsymbol{x}_{p_{\text {tumor }}^{0}}-\delta \boldsymbol{n}_{p_{\text {tumor }}^{0}} & \delta \ll r_{0}
\end{array}
$$

where $\boldsymbol{n}$ denotes the normal vector of the surface. The new particle is pushed inside the tissue to ensure that it will never fall outside the endometrium. As $\delta \ll r_{0}$ the following operations will push the new particle further into the tissue.

In case of a myoma, all tumor particles are equally able to divide. Thus a tumor particle $p_{\text {tumor }}^{i}$ is randomly selected and divided:

$$
\begin{aligned}
p_{\text {tumor }}^{i} & \rightarrow p_{\text {tumor }}^{i}+p_{\text {tumor }}^{n+1} \quad i=\operatorname{RAND}(0, n) \\
\mathcal{R}_{\text {dividemyoma }}: \boldsymbol{x}_{p_{\text {tumor }}^{n+1}} & =\boldsymbol{x}_{p_{\text {tumor }}^{i}}+0.5 \boldsymbol{r}_{0} \\
\boldsymbol{x}_{p_{\text {tumor }}^{i}}^{\prime} & =\boldsymbol{x}_{p_{\text {tumor }}^{i}}-0.5 \boldsymbol{r}_{0}
\end{aligned}
$$

The vector $\boldsymbol{r}_{0}$ is uniformly distributed over the surface of the sphere with radius $r_{0}$. Details about the computation of such a vector can be found in reference [6]. The random displacement ensures a global spherical growth pattern while using only local operations.

Adapt tissues. The new particle as well as the fairing of the surface in the previous iteration change the force felt by almost all particles. Each particle accumulates the forces acting on itself from the subset of close enough particles, that is $r_{i j} \leq \frac{3}{2} r_{0}$ :

$$
\boldsymbol{F}_{p^{i}}^{t}=\sum_{j \in \mathcal{N}} F^{t}\left(r_{i j}\right) \frac{\boldsymbol{r}_{i j}}{\left|\boldsymbol{r}_{i j}\right|}
$$

Based on the force, the particle is translated using equation 11. The time step $h$ has to be selected small enough to prevent particles from outrunning each other. Naturally the positions would only be updated after all particles have accumulated their respective forces. For small changes, i.e. small values of $h$, the convergence is faster using serial computation, that is every particle $p^{i}$ is updated based on equations 2 and 1 and following particles $p^{j}, j>i$ are updated based on the new positions $\boldsymbol{x}_{p^{i}}^{t+1}$.

Adapt surface. As the endometrium is always covering all tissues, its shape is adapted according to the tumor's deformation. All particles apply forces on the surface, again depending on the distance to it. In this processing step it is crucial that exact distances from the particle to the surface and not just to the particles on the surface are computed. This measure prevents penetrations through the surface. The resulting force on a triangle $\mathcal{T}$ is transferred to the three vertices of $\mathcal{T}$. After the accumulation of the external forces, the surface springs are relaxed. As for the particles, the stationary equilibrium of all springs is computed. 
Surface fairing. Without provisions, irregularities emerge easily since the surface is deformed in almost arbitrary directions. Adaptive subdivision is introduced to mimic the actual growth of the endometrium. The set of triangles $\mathcal{S} \subset \mathcal{M}$ with an area larger then a threshold $t$ is subdivided using quaternary subdivision. The set $\mathcal{S}_{\text {adj }} \subset \mathcal{M}, \mathcal{S}_{\text {adj }} \cap \mathcal{S}=\emptyset$ of triangles $\mathcal{T}_{\text {adj }}$ adjacent to any triangle $\mathcal{T}_{\text {subdiv }} \in \mathcal{S}$ is subdivided to ensure a correct triangulation of the complete mesh $\mathcal{M}$ :

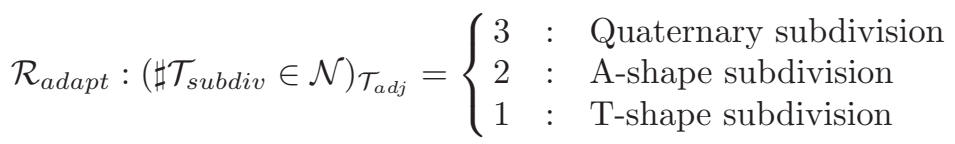

where $\mathcal{N}$ is the set of neighbors of a triangle. Image a) in Figure 1 illustrates the different topological operations.

Following the subdivision, the quality of the triangles is enhanced by edge swapping. The ratio of the inscribed to the circumscribed circle radii is used as quality measure, as it is faster to compute than other measurements and has been shown to have only one stationary value for the equilateral triangle [9]: $\rho_{\mathcal{T}}=\frac{r_{\text {in }}}{r_{\text {circum }}}$. For any two neighboring triangles, the common edge is flipped if $\rho_{\mathcal{T}_{1}^{\prime}}+\rho_{\mathcal{T}_{2}^{\prime}}>\rho_{\mathcal{T}_{1}}+\rho_{\mathcal{T}_{2}}$. It should be noted that this operation has a great impact on the regularity of the overall mesh $\mathcal{M}$.

Finally, small triangles are removed by collapsing the shortest edge of the triangle. To ensure topological correctness, this operation is only applied in a conservative manner, i.e. if the involved vertices as well as their common neighbor vertices all have valences larger three [10].

After fairing of the surface, all springs are assigned a new equilibrium length corresponding to the current length and all external forces are deleted. This concludes the transition into a new stationary equilibrium of the growth process.

\section{Results and Discussion}

The algorithm presented produces highly satisfactory results. The grown polyps are, on a macroscopic level, very similar to real cases, as has been attested to experienced gynecologists. Figure 1 shows an example of a polyp after 190 growth iterations (b), 290 respectively (c). More images and animations of the growing process can be found online at www.ee.ethz.ch/ rsierra/Miccai03.

The surface fairing and the mass-spring paradigm might seem somewhat complex. At first glance, it would seem appropriate to seek an alternative approach where the mass-spring system can be replaced, e.g. by using surface particles [14]. Two reasons speak for the use of a continuous surface representation. As mentioned, an exact distance computation is mandatory to prevent particles from penetrating the surface. In case of particle use only, the density of particles required on the surface would explode beyond any reasonable computational limit. The incorporation of a surface representation renders a final embedding of the resulting pathology into the organ model unnecessary, an additional operation that is required in the previously presented methods. Thus, the selected representation of the surface leads to a simpler and faster computation. 
Collisions of the pathology's surface with the uterine cavity can easily be handled similarly to the third step of the algorithm by assigning a purely repulsive force profile to the cavity's surface.

The interaction between inner particles and the surface ensures a controlled growth pattern. Without the surface, the tumor growth would generate a random pattern in space as the inter-particle attraction is not sufficient to guide the growth process. The small tension in the surface introduced by the spring stiffness also acts as a smoothing factor: without this tension, the endometrium would follow every perturbation of the outermost particle layer leading to surfaces with very high frequencies that do not look realistic. Too large tensions would result in an extremely stiff surface that may even collapse. Nevertheless, once an appropriate value is chosen, it can be used for all experiments.
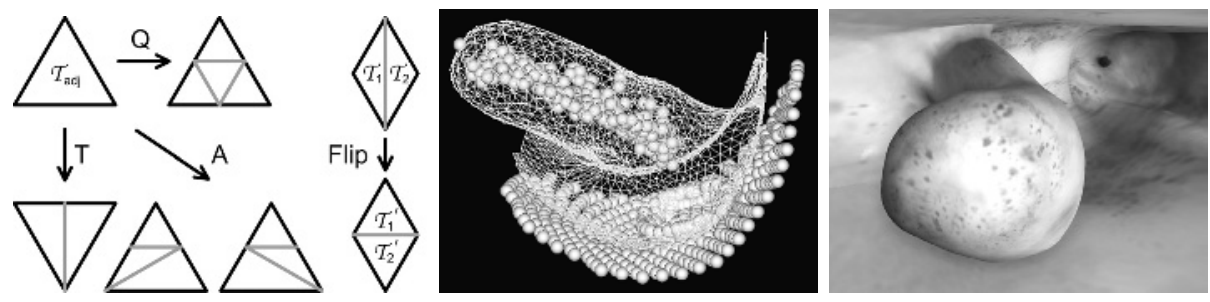

Fig. 1. a) Triangle operations. b) Inner and c) surface view of a polyp.

The computational time required is significantly larger than the one needed for the previous approaches. However, the pathologies can be generated offline before the actual training since the algorithm is autonomous. In addition, standard particle system optimization techniques can be implemented to reduce the computational time.

The benign tumors implemented are a common cause of bleeding disorders, dysmenorrhea and infertility, and account for most of hysteroscopic interventions. Despite the amount of research, the exact etiology of these pathologies remains unknown. To our knowledge, there are no publications that investigate exact metrics of the introduced pathologies beyond the gross description provided, that could be used for validation. Medical literature in the field focuses on microscopic analysis of tissue samples for the determination of the malignancy of the tumor. So far, intraoperative images remain as the only means for further analysis of the pathologies.

Several recordings of hysteroscopic interventions have been studied to estimate the phenomenological properties, such as curvatures or length ratios. A reconstruction of three dimensional measurements from the images is exacerbated by the non-rigidness of the objects and related to the existence of sufficient visible texture on the surface. In addition, the limited field of view and the uncalibrated and highly distorted camera render an exact determination of single values extremely difficult. We are currently forced to roughly estimate the metrics with the help of expert knowledge, as we are still in the process of collecting appropriate intraoperative recordings. 


\section{Conclusion and Future Research}

The new approach for the generation of pathologies in virtual organs for surgical training simulators shows many advantages compared to previous growth models. Different pathology types can directly be generated into the organ model using a single coherent framework, that is based on biological knowledge to grow the desired structures. As the process is independent of the organ, it can directly be used to generate pathologies in any other organ, e.g. colon polyps.

There are a number of avenues for future research. More biological knowledge can be incorporated into the model, ranging from competing cell cultures for malign tumors to the influence of vascularization on the shape. Provided the elastic properties of the endometrium are known, a finite element representation of the surface can be implemented and compared to the current surface representation. Once convenient intraoperative recordings are available, 3D computer vision techniques will be employed to extract precise metrics of the pathologies. The algorithm can then be tuned to match the additional knowledge.

Acknowledgments. This work has been supported by the NCCR CO-ME of the Swiss National Science Foundation.

\section{References}

1. T. Cootes et al. Active Shape Models - Their Training and Application. Computer Vision and Image Understanding, 61(1): 38-59, 1995.

2. G. Cottet and P. Koumoutsakos. Vortex Methods - Theory and Practice. Cambridge University Press, New York, 2000.

3. C. Jamora and E. Fuchs. Intercellular adhesion, signalling and the cytoskeleton. Nature Cell Biology, 4: 101-108, 2002.

4. A. Kelemen et al. Elastic Model-Based Segmentation of 3-D Neororadiological Data Sets. IEEE Transactions on Medical Imaging, 18(10): 828-839, 1999.

5. R. Kurman and M. Mazur. Blaustein's Pathology of the Female Genital Tract. Springer, Berlin, New York, fourth edition, 1990.

6. G. Marsaglia. Choosing a Point from the Surface of a Sphere. Ann. Math. Stat., 43: 645-646, 1972.

7. K. Montgomery et al. Surgical Simulator for Hysteroscopy: A Case Study of Visualization in Surgical Training. IEEE Visualization 2001, 2001.

8. F. Netter. Farbatlanten der Medizin, Band 3: Genitalorgane. Georg Thieme Verlag, Stuttgart, New York, second edition, 1987.

9. P. Pébay and T. Baker. A Comparison of Triangle Quality Measures. 10th International Meshing Roundtable, Proceedings: 327-340, 2001.

10. M. Quicken et al. Parameterization of closed surfaces for parametric surface description. In CVPR, volume 1, pages 354-360, June 2000.

11. R. Sierra, M. Bajka, and G. Székely. Pathology Design for Surgical Training Simulators. In Proceedings IS4TM, 2003.

12. R. Sierra, G. Székely, and M. Bajka. Generation of Pathologies for Surgical Training Simulators. MICCAI, Proceedings, Part II: 202-210, 2002.

13. G. Székely et al. Virtual Reality-Based Simulation of Endoscopic Surgery. Presence, 9(3): 310-333, 2000.

14. D. L. Tonnesen. Dynamically Coupled Particle Systems for Geometric Modeling, Reconstruction, and Animation. PhD thesis. 